\title{
Aplicación de la Clase Inversa en la Enseñanza de la Electrónica Analógica en un Grupo de Alto Rendimiento Académico
}

\author{
Beatriz Trenor ${ }^{\mathrm{a}}$, Gema Prats-Boluda ${ }^{\mathrm{a}}$ Y Yiyao Ye Lin ${ }^{\mathrm{a}}$
}

${ }^{a}$ Universitat Politècnica de València. Departamento de Ingeniería Electrónica (btrenor@eln.upv.es; geprabo@eln.upv.es; yiye@eln.upv.es).

\begin{abstract}
The need of increasing the participation of the student in the teaching and learning process at University has led to the concept of flip teaching. This methodology is characterized by the fact that the student works on the theoretical concepts independently and using mainly audiovisual materials. Later, the student works on applied activities in the teaching room under the teacher supervision, in order to reinforce his learning. The objective of the present study is to evaluate the impact of flip teaching in analog electronics in the Degree of Engineering in Electronics and Automatics. This methodology has been applied during the academic years 2014-2015, 20152016, and 2016-2017 in the group of high academic performance (ARA group), in which English is the medium of instruction. 95\% of our students considered that flip teaching was a very adecuate methodology for the teaching and learning process of this course. Furthermore, the evaluation methods based on the tool SOCRATIVE have been positevely accepted. Finally, the academic performance was higher in the ARA group with respect to the rest of the groups, which did not happen in previous years when no flip teaching was applied at all.
\end{abstract}

Keywords: Flip teaching, high academic performance group, Polimedia, SOCRATIVE.

\footnotetext{
Resumen

De la necesidad de una mayor participación del alumnado universitario en el proceso de enseñanza-aprendizaje ha surgido el concepto de la docencia inversa. Mediante el uso de esta metodología, el alumno trabaja, de manera independiente, los conceptos más teóricos, con la ayuda de material audiovisual principalmente. Posteriormente, en clase, el alumno realiza actividades más prácticas dirigidas por el profesor y así afianza los conceptos aprendidos. El objetivo del presente trabajo es evaluar el impacto de dicha metodología en la enseñanza de la electrónica analógica en la titulación de Grado en Ingeniería Electrónica y Automática. Se ha aplicado la docencia inversa en los cursos 2014-2015, 2015-2016 y 2016-2017 en el grupo de alto rendimiento académico, que cursa la asignatura en inglés. La valoración del alumnado ha sido muy positiva: un $95 \%$ del alumnado considera muy adecuada la metodología. Además, los métodos de evaluación mediante el uso de la herramienta SOCRATIVE se han valorado positivamente y el rendimiento académico del grupo ARA ha mejorado con
} 
respecto a los demás grupos, lo cual no sucedió en años anteriores cuando no se aplicaba la docencia inversa.

Palabras clave: Clase Inversa, Grupo de alto rendimiento académico, Polimedia, SOCRATIVE.

\section{Introducción}

La docencia tradicional se basa en las lecciones magistrales donde la pasividad del alumno ralentiza su proceso de aprendizaje. La adaptación al espacio europeo de educación superior (EEES) de la educación universitaria en España ha permitido la introducción y fomento de metodologías más activas en el proceso de enseñanza-aprendizaje en las aulas. Especialmente, para las asignaturas más prácticas, es necesario que el alumno se involucre y asuma su responsabilidad en el proceso de aprendizaje. Debido a estas necesidades y al auge de las nuevas tecnologías audiovisuales se va instaurando en docencia la clase inversa o flipped classroom. Esta metodología ha sido fuertemente influenciada por la estrategia educativa de Peer Instruction y la enseñanza just-in-time (Mazur 1996; Santiago y Díez). Mediante este tipo de docencia, el alumno, de manera individual y responsable, adquiere los conceptos más teóricos con el material (principalmente audiovisual) proporcionado por el profesor. Posteriormente, en las sesiones presenciales con el profesor, en el aula, los alumnos pueden plantear sus dudas y centrarse en las tareas de aplicación más prácticas de la asignatura. Desde el punto de vista de la pirámide de aprendizaje de Bloom definida en 1956 y revisada en 2000 (Bloom 1956; Eduteka), la clase inversa fomenta que el alumno realice las tareas de la base (comprender y recordar) de manera independiente y en el aula se centre en las tareas más altas de la pirámide (crear, evaluar, analizar, aplicar).

El concepto de flipped teaching fue introducido en 2007 por los profesores Sams y Bergmann del Instituto Woodland Park en Colorado, al proponer un sistema de recuperación de clases mediante grabaciones de vídeo. Los resultados de esta experiencia fueron muy positivos (Sams y Bergmann, 2013) y esta metodología se está extendiendo a nivel mundial.

El impulso de esta iniciativa en la Universitat Politècnica de València desde el Vicerrectorado de las Tecnologías de la Información y de las Comunicaciones y el Instituto de Ciencias de la Educación en los últimos años, nos llevó a los profesores de la asignatura de Electrónica Analógica (titulación de Grado en Ingeniería Electrónica Industrial y Automática en la Escuela Técnica Superior de Ingeniería del Diseño) a aplicarla en clase.

Para aplicar la metodología de la clase inversa, quisimos hacerlo en uno de los 3 grupos de la asignatura, en el grupo menos numeroso, para poder llevar un seguimiento más apropiado. El grupo menos numeroso es el grupo de alto rendimiento académico (grupo ARA) en el cual la docencia se imparte en inglés. Por este motivo, las grabaciones de los elementos Polimedia se realizaron en inglés.

Una de las mayores dificultades que encontramos los profesores de la aplicación de esta metodología estaba relacionado con la evaluación. Por ello, decidimos emplear diversos métodos de evaluación capaces de motivar al alumnado. En concreto empleamos una

(cc) EY-NC-ND 2017, Universitat Politècnica de València 
herramienta para realizar tests en línea desde los teléfonos móviles, llamada SOCRATIVE (SOCRATIVE), ya que el empleo de las nuevas tecnologías para la evaluación parece motivar más al alumno.

\section{Objetivos}

El objetivo principal del presente trabajo es la aplicación de la docencia inversa para el proceso de enseñanza-aprendizaje de la asignatura de electrónica analógica en un grupo de alto rendimiento académico. Para ello, se han empleado grabaciones polimedia, realización de cuestiones y tests en línea mediante la herramienta SOCRATIVE. Otro objetivo del presente trabajo era valorar el grado de aceptación de esta metodología por parte del alumnado y si la perciben como una carga adicional excesiva o no, así como la mejora en su rendimiento académico.

\section{Desarrollo de la Innovación}

La experiencia de docencia inversa descrita en el presente trabajo se desarrolla en el contexto de la asignatura de Electrónica Analógica, asignatura troncal de segundo curso (cuatrimestre B) de la titulación Grado en Ingeniería Electrónica Industrial y Automática, en la Escuela Técnica Superior de Ingeniería del Diseño. Se trata de una asignatura con un gran número de alumnos (entre 150 y 175) divididos en tres grupos. El grupo en el cual se ha realizado la clase inversa es el grupo ARA, cuya docencia se desarrolla en inglés. Dicho grupo cuenta con un número de alumnos de 34 en el presente curso (2016-2017), 24 en el curso anterior (2015-2016) y 23 alumnos en el curso 2014-2015. Además hay dos grupos más, un grupo de mañana y un grupo de tarde, que cursan la asignatura en castellano y son grupos más numerosos (entre 65 y 75 alumnos). El temario de la asignatura es amplio y complejo y requiere la realización de muchos ejercicios prácticos para una comprensión profunda de la asignatura. Por ello, con el objetivo de dejar más tiempo en clase a la realización de ejercicios que afianzan los conocimiento adquiridos en teoría, los profesores de la asignatura planteamos la posibilidad de emplear la metodología de la docencia inversa. Para comenzar con esta experiencia, nos centramos en el grupo más reducido (grupo ARA) y así realizar un mejor seguimiento. A continuación se describe la metodología empleada en el curso 2014-2015 y mejorada en los cursos 2015-2016 y 20162017.

La metodología docente empleada en la asignatura, aunque tradicional, se ha adaptado progresivamente al espacio europeo de educación superior (EEES) e incluye diversas actividades y sistemas de evaluación. Los alumnos disponen de las diapositivas empleadas en las clases de teoría, de diversas colecciones de problemas para resolver en casa y en clase, así como de exámenes resueltos de años anteriores. Además, los alumnos disponen de documentos para la preparación y realización de las prácticas de laboratorio. La evaluación consta de dos pruebas parciales y una final en la que se evalúan los conocimientos adquiridos en las prácticas de laboratorio (preguntas tipo test que 
constituyen un $25 \%$ de la nota final), los conceptos teóricos y resolución de problemas (cuestiones de respuesta abierta que constituyen un $60 \%$ de la nota final). Finalmente, los alumnos realizan en grupos de tres un proyecto de diseño y simulación que exponen oralmente en clase para mejorar sus habilidades de documentación y presentación oral (15\% de la nota final).

Las técnicas de docencia inversa se han introducido en el grupo ARA, mediante el uso de 11 elementos polimedia, vídeos grabados en inglés con el contenido de 2 de los 8 temas de la asignatura. La teoría del tema 3 y el tema 7, 2 temas cruciales en la asignatura, está explicada en los 11 vídeos. En la programación diaria de la asignatura, a la cual los alumnos tienen acceso y se muestra una parte en el anexo 1, se indica los vídeos que tienen que ver los alumnos antes de asistir a clase. Además, los alumnos deben resolver en casa unas cuestiones sencillas acerca de los vídeos visualizados para afianzar su comprensión. En clase, los propios alumnos y/o el profesor corrigen en la pizarra dichas cuestiones. Durante la corrección los alumnos plantean sus dudas y el profesor las resuelve. A continuación, los alumnos realizan en línea unas preguntas tipo test, mediante el uso de la aplicación SOCRATIVE desde sus teléfonos móviles. Las notas obtenidas en dichos tests (registradas mediante el software SOCRATIVE en una hoja excel) podrán suponer hasta un punto adicional en la nota final de la asignatura. Cabe destacar que la resolución de las cuestiones y las preguntas del test las realiza el alumno con una mejor comprensión de los conceptos teóricos puesto que ha visualizado previamente los vídeos en una ambiente más distendido que en clase, pudiendo retroceder si algo no le ha quedado claro. Otra ventaja clara es un aumento del tiempo dedicado en clase a la resolución posterior de ejercicios y problemas más prácticos. Durante las clases presenciales de los temas 3 y 7, la mayor parte del tiempo los alumnos han trabajado en la resolución de ejercicios activamente con el apoyo del profesor.

En el curso 2014-2015, los alumnos disponían de los vídeos correspondientes al tema 3 únicamente. Al finalizar el curso, se les pasó la encuesta del anexo 2, y dado que la aceptación fue muy positiva (ver sección de resultados) se decidió ampliar el material de polimedia grabando vídeos del tema 7 para los cursos 2015-2016 y 2016-2017. La introducción de la herramienta SOCRATIVE ha tenido lugar durante el presente curso 2016-2017 y los alumnos han realizado asimismo una encuesta de 4 preguntas en línea acerca de esta herramienta, vía PoliformaT (ver sección de resultados).

\section{Resultados}

En esta sección se describen los resultados de la aplicación de la metodología de docencia inversa en los cursos 2014-2015, 2015-2016 y 2016-2017.

En los 3 cursos, durante la primera clase del tema 3, en la que los alumnos debían traer las cuestiones resueltas y haber visualizado 2 de los vídeos, el profesor observó que prácticamente todos los alumnos habían visto los vídeos y traían las cuestiones resueltas. Algún alumno admitió honestamente que no había visto el vídeo por despiste o por falta de tiempo. Por tanto, un primer e importante resultado positivo fue conseguir que

(c) EY-NC-ND 2017, Universitat Politècnica de València 
prácticamente la totalidad del alumnado asistiera a clase con buenos conocimientos del tema a trabajar durante la sesión presencial. El profesor hace un breve sondeo oral acerca de los vídeos y los alumnos muestran una aceptación inicial, comentando que han podido ver los vídeos con tranquilidad y retrocediendo en algunos casos. En los vídeos, el profesor utiliza las diapositivas del tema (accesibles en poliformaT) y además realiza cálculos sencillos en una Tablet que se pueden seguir con claridad en el vídeo, como si fuera la pizarra de un aula.

Durante la corrección de las cuestiones, los alumnos plantean numerosas dudas o dificultades que han tenido para resolverlas, afianzando así los conceptos trabajados. Posteriormente, durante la resolución de ejercicios prácticos por parte de los alumnos en clase, el profesor se pasea por las mesas resolviendo dudas a medida que van surgiendo. La implicación del alumnado es muy significativa. Puesto que el grupo no es muy numeroso, el profesor puede dedicar el tiempo necesario para atender correctamente a los alumnos durante estas sesiones.

Al finalizar los cursos 2014-2015 y 2015-2016, un total de 17 y 20 alumnos, respectivamente, respondieron a la encuesta mostrada en el anexo 2 , y cuyas respuestas se describen en la Figura 1.

Como muestra la Figura 1, los alumnos han asumido la responsabilidad de visualizar los vídeos y en general opinan que les ha supuesto un ahorro de tiempo puesto que afianzan los conceptos de manera más efectiva. Los vídeos les han parecido fáciles de seguir, excepto a un alumno en el curso 2015-2016. En cuanto al ritmo, parece que en el curso 2015-2016 $45 \%$ de los alumnos piensan que es demasiado lento. Quizás el ritmo de los vídeos del tema 7 que no visualizaron los alumnos del curso anterior era demasiado lento. Esta observación será de ayuda para intentar grabar vídeos de los capítulos restantes a un ritmo más rápido. Las explicaciones realizadas en la Tablet durante los vídeos, similares a las que hace el profesor en la pizarra, también han tenido una gran aceptación. En el curso 2015-2016 un $45 \%$ de los alumnos han visualizado los vídeos sin detenerse, posiblemente porque el ritmo fuera lento para ellos. En cuanto a las cuestiones resueltas en casa y corregidas en clase acerca de los vídeos han resultado ser difíciles para un $12 \%$ y $40 \%$ en los cursos 2014 2015 y 2016-2016, respectivamente. Finalmente, un 95\% de los alumnos en los 2 cursos están de acuerdo con el uso de esta metodología y un $60 \%$ de los alumnos desearía tener vídeos para todos los temas de la asignatura. Por este motivo, los profesores de la asignatura vamos a ampliar el número de grabaciones para futuros cursos.

En esta encuesta los alumnos añadieron opiniones y sugerencias personales entre las cuales nos gustaría destacar las siguientes: i) los vídeos iniciales eran demasiados largos. En efecto la duración aproximada de los vídeos del tema 3 son de 20 minutos, y posteriormente se siguienron las indicaciones de la UPV para grabar vídeos Polimedia de 10 minutos en el tema 7. ii) Los alumnos preferirían resolver en clase más ejercicios, para lo cual se debería incrementar la práctica de la clase inversa mediante grabación de más vídeos.

En el presente curso 2016-2017, los alumnos realizan las preguntas tipo test mediante la aplicación SOCRATIVE en línea en sus teléfonos móviles o en los ordenadores de clase. Las notas obtenidas son muy altas, obteniendo unas medias de $87 \%$ y $85.6 \%$ en los 2 tests 
Aplicación de la Clase Inversa en la Enseñanza de la Electrónica Analógica en un Grupo de Alto Rendimiento Académico

correspondientes a los vídeos del tema 3 , y unas medias de $80 \%, 80.6 \%$ y $93.1 \%$ en los 3 tests correspondientes a los vídeos del tema 7. Las notas elevadas muestran que los alumnos han adquirido correctamente los conceptos teóricos básicos y han visualizado los vídeos con atención. En la tabla 1 se resume la opinión de los alumnos acerca del uso de la herramienta SOCRATIVE para la evaluación de los conocimientos adquiridos a través de los vídeos.

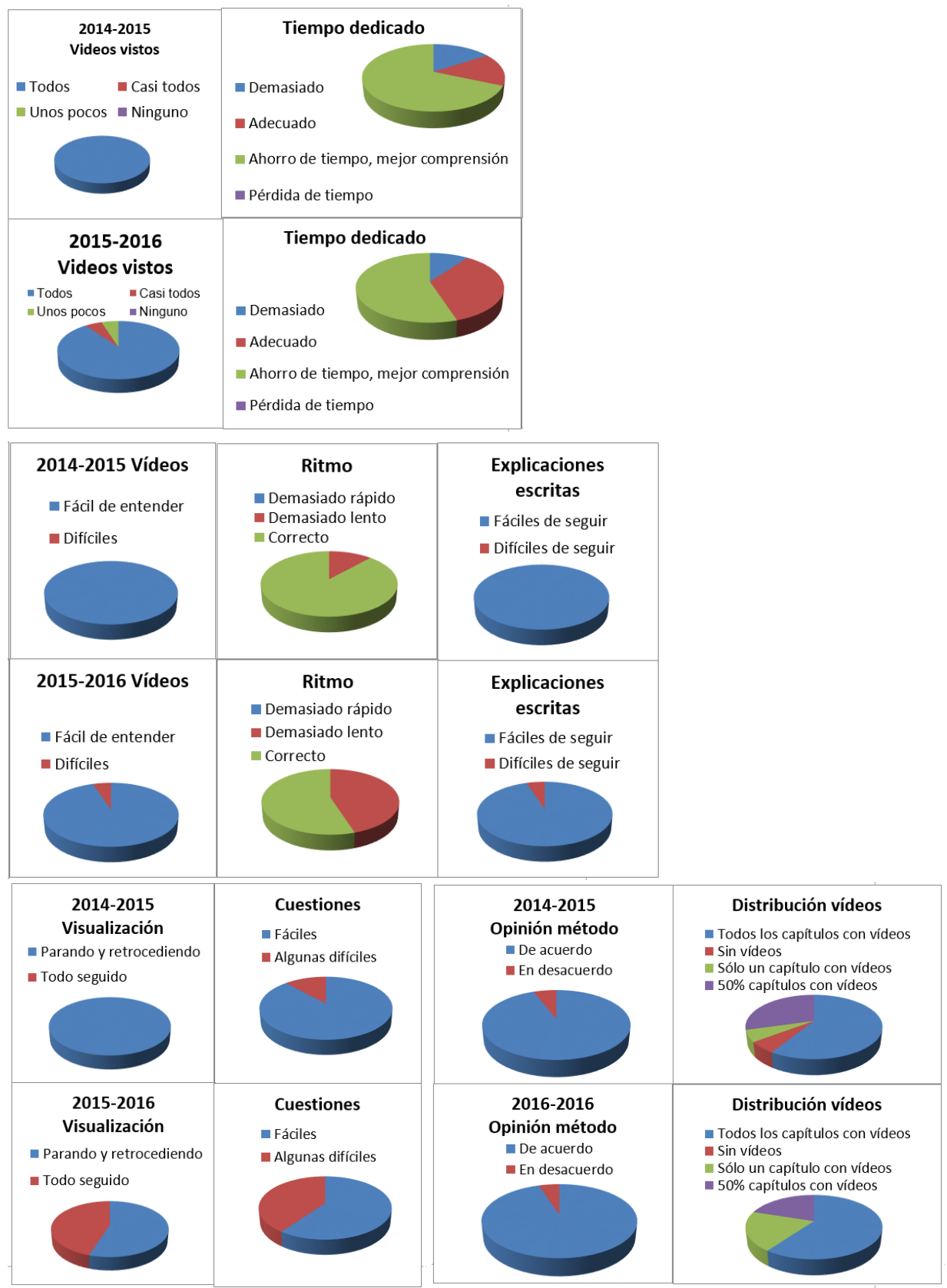

Fig. 1 Resultado de la encuesta (ver anexo 2) realizada por el alumnado en los curso 2014-2015 y 2015-2016.

(cc) EY-NC-ND 2017, Universitat Politècnica de València 
Tabla 1. Resultados de la encuesta acerca de SOCRATIVE

1. Los cuestionarios realizados en clase con la aplicación SOCRATIVE me han parecido adecuados para evaluar su comprensión de los vídeos

\begin{tabular}{|c|c|c|c|c|}
\hline $\begin{array}{c}\text { Totalmente en } \\
\text { desacuerdo }\end{array}$ & En desacuerdo & NS/NC & De acuerdo & Totalmente de acuerdo \\
\hline 0 & 1 & 1 & 10 & 7 \\
\hline $\mathbf{2 .}$ Las preguntas de la herramienta SOCRATIVE me han servido de motivación para ver los vídeos \\
\hline $\begin{array}{c}\text { Totalmente en } \\
\text { desacuerdo }\end{array}$ & En desacuerdo & NS/NC & De acuerdo & Totalmente de acuerdo \\
\hline 0 & 5 & 3 & 10 & 1 \\
\hline
\end{tabular}

3. La herramienta Socrative me parece una herramienta adecuada para la evaluación

\begin{tabular}{|c|c|c|c|c|}
\hline $\begin{array}{c}\text { Totalmente en } \\
\text { desacuerdo }\end{array}$ & En desacuerdo & NS/NC & De acuerdo & Totalmente de acuerdo \\
\hline 0 & 1 & 0 & 15 & 3 \\
\hline
\end{tabular}

4. Las preguntas de la herramienta SOCRATIVE se adecuan al contenido de los vídeos

\begin{tabular}{|c|c|c|c|c|}
\hline $\begin{array}{c}\text { Totalmente en } \\
\text { desacuerdo }\end{array}$ & En desacuerdo & NS/NC & De acuerdo & Totalmente de acuerdo \\
\hline 0 & 0 & 1 & 9 & 9 \\
\hline
\end{tabular}

De los resultados de la encuesta se deduce que el hecho de tener un cuestionario no sea suficiente motivación para ver los vídeos. En nuestra opinión, la propia responsabilidad que se deposita en los alumnos les motiva para ver los vídeos. Además pueden comprobar que si no lo ven no podrán seguir las clases. En general los alumnos están de acuerdo con este método para evaluar la comprensión de los vídeos. Debe destacarse que sólo 19 alumnos han rellenado la encuesta y por tanto las conclusiones extraídas podrían ser más sólidas si hubieran contestado los 34 alumnos. Esta encuesta se hizo a través de PoliformaT y quizás los alumnos no eran conscientes de la fecha límite para rellenarla.

Finalmente, considerando los resultados académicos obtenidos en el curso 2015-2016 se puede realizar una comparación entre el grupo con docencia inversa y el resto de grupos. Cabe destacar que la comparación realizada tiene ciertas limitaciones que comentaremos a continuación, pero puede servir como un indicio de que la metodología aplicada es beneficiosa.

En la tabla 2 se muestran las medias y desviaciones típicas de las notas del grupo ARA y del resto de grupos en los cursos 2013-2014, 2014-2015 y 2015-2016. Además, puesto que las notas no siguen una distribución normal, se muestran también las medianas y los diagramas de caja (Box plot) en la Figura 2. 
Tabla 2. Resultados Académicos de los distintos cursos y grupos.

\begin{tabular}{|c|c|c|c|c|}
\hline $\begin{array}{c}\text { Curso } \\
\text { Académico }\end{array}$ & Grupo & Media & $\begin{array}{c}\text { Desviación } \\
\text { típica }\end{array}$ & Mediana \\
\hline \multirow{2}{*}{$2013-2014$} & ARA & 6.46 & 1.51 & 6.1 \\
\cline { 2 - 5 } & Resto de grupos & 6.86 & 1.78 & 7 \\
\hline \multirow{2}{*}{$2014-2015$} & ARA & 5.78 & 2.06 & 5.81 \\
\cline { 2 - 5 } & Resto de grupos & 5.38 & 2.19 & 5.69 \\
\hline \multirow{2}{*}{$\mathbf{2 0 1 5 - 2 0 1 6}$} & ARA & $\mathbf{6 . 3 6}$ & $\mathbf{1 . 3 7}$ & $\mathbf{5 . 9 5}$ \\
\cline { 2 - 5 } & Resto de grupos & $\mathbf{5 . 2 9}$ & $\mathbf{1 . 7 5}$ & $\mathbf{5 . 4}$ \\
\hline
\end{tabular}

Si realizamos una comparación estadística de las medias de los distintos grupos, siguiendo el test t-student, la diferencia de medias entre el grupo ARA y el resto de grupos es significativa $(\mathrm{p}<0.05)$ únicamente en el curso 2015-2016.

Puesto que la distribución de notas no sigue una variable normal y el tamaño de muestra no es grande, se ha realizado también el test Wilcoxon. El resultado obtenido ha sido que las medianas del grupo ARA y el resto de grupos pueden considerarse iguales con una probabilidad de 0.2593 , 0.4558 y 0.0137 en los cursos 2013-2014, 2014-2015 y 2015-2016, respectivamente. Por tanto, puede considerarse que las medianas son significativamente distintas ( $p<0.05)$ únicamente en el curso 2015-2016. 

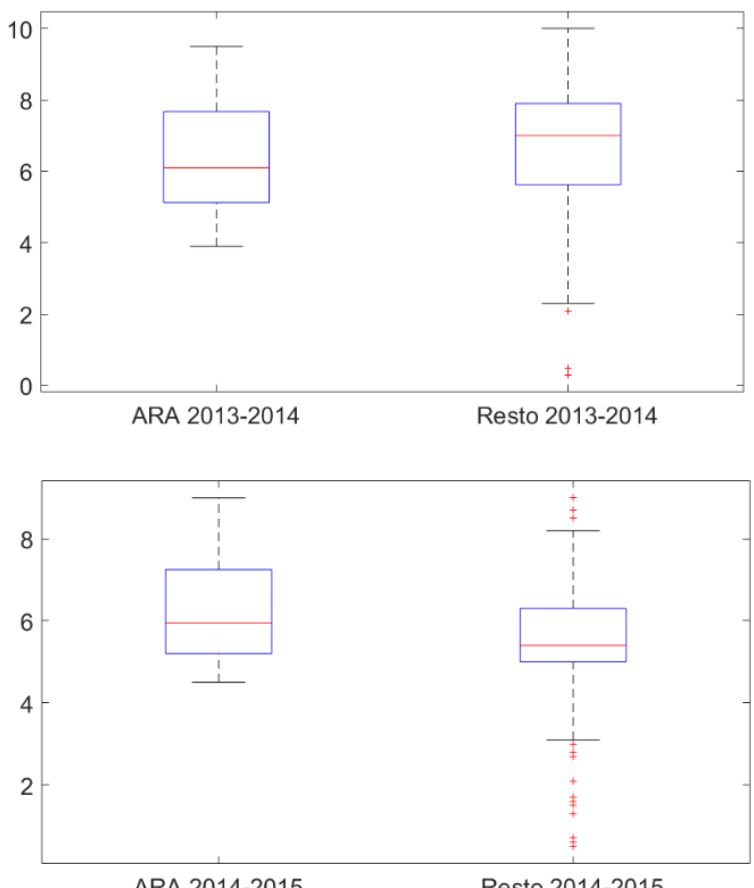

ARA 2014-2015

Resto 2014-2015

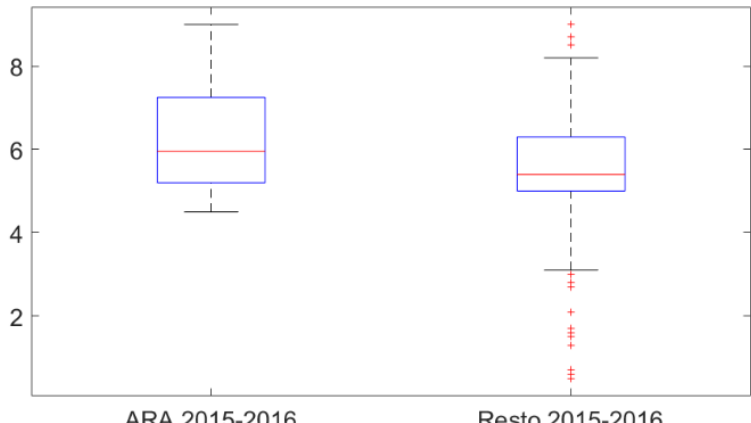

Fig. 2 Resultados académicos de los distintos grupos y cursos académicos representados en diagramas de caja (Box plot).

Como limitaciones de esta comparación debe considerarse que la docencia inversa se ha empleado en 2014-2015 en un solo tema (tema 3) de 8 temas que tiene la asignatura y en 2015-2016 en dos temas (tema 3 y tema 7). Sin embargo, el tema 3 y el tema 7 son 2 de los temas más importantes de la asignatura en los que se introducen conceptos que aparecen en todos los ejercicios de la asignatura. Son concepto difíciles de afianzar.

Por otra parte, los alumnos del grupo ARA, en general, tienen una motivación especial. Son alumnos que están dispuestos a cursar la asignatura en inglés a pesar de su dificultad y que en general pueden tener resultados académicos mejores. Sin embargo, en el curso 20132014, cuando no se aplicaba la metodología de clase inversa, la nota media no era superior en el grupo ARA, por tanto no siempre son alumnos con mejores resultados académicos.

(c) ) EY-NC-ND 2017, Universitat Politècnica de València 
Por supuesto, hay muchos otros factores que influyen en los resultados, como por ejemplo el hecho de que el profesorado en los distintos grupos no es el mismo.

\section{Conclusiones}

Los alumnos y el profesorado valoran positivamente la aplicación de la docencia inversa en el proceso de enseñanza-aprendizaje de la electrónica analógica. El 95\% de los alumnos están de acuerdo con el uso de esta metodología y un $60 \%$ de los alumnos desearía tener vídeos para todos los temas de la asignatura. Las cuestiones resueltas en casa y corregidas en clase así como el uso de la herramienta SOCRATIVE (test en línea) son herramientas válidas para la evaluación de la clase inversa. Esta metodología de trabajo permite mejorar el rendimiento académico y la satisfacción de los alumnos en su proceso de aprendizaje.

\section{Referencias}

BLOOM, B. S. and KRATHWOHL, D. R. (1956). Taxonomy of Educational Objectives: The Classification of Educational Goals. Handbook I: Cognitive Domain. New York: Longmans Green

EDUTEKA. Taxonomía de Bloom para la era digital. <http://eduteka.icesi.edu.co/articulos/TaxonomiaBloomDigital $>$ [Consulta: 28 Marzo 2017]

MAZUR, E. (1996) Peer Instruction: A User's Manual, New York: Prentice Hall.

SAMS, A. y BERGMANN, J. (2013). "Flip Your Students' Learning”. Educational Leadership, 70(6), 16-20.

SOCRATIVE, by Master Connect <https://www.socrative.com/> [Consulta : 28 Marzo 2017].

SANTIAGO, R. Y DIEZ, A. The flipped classroom. <http://www.theflippedclassroom.es/metodologia-teaching-methods/> [Consulta: $21 \mathrm{de}$ marzo de 2016] 
ANEXO 1.

PROGRAMACIÓN DIARIA DE LA ASIGNATURA PARA LOS ALUMNOS. MES

\begin{tabular}{|c|c|c|}
\hline & Monday (8:30-10:30) & Tuesday (11:30-14:30) \\
\hline & - LECTURE - & - LECTURE / LABORATORY - \\
\hline 30 & $\begin{array}{l}\text { Presentation + Projects } \\
\text { CHAPTER 1. INTRODUCTION } \\
\text { (total } 1 \mathrm{~h} \text { ) } \\
\text { EXERCISES }\end{array}$ & $\begin{array}{ll}1 & \\
\text { CHAPTER 2. ANALOG SIGNALS } \\
\text { (total } 3 \mathbf{h})\end{array}$ \\
\hline 6 & NO CLASS & $\begin{array}{l}7 \\
\text { LABORATORY EXP. 1: Instruments and } \\
\text { diode circuits } \\
\text { Previous calculations }\end{array}$ \\
\hline 13 & $\begin{array}{l} \\
\text { CHAPTER 3. ANALOG } \\
\text { SYSTEMS (total 9h) } \\
\text { Watch videos } 1 \text { and } 2 \\
\text { Questions videos } 1 \text { and } 2\end{array}$ & \begin{tabular}{|l}
14 \\
CHAPTER 3. ANALOG SYSTEMS \\
Watch videos 3 and 4 \\
Questions videos 3 and 4
\end{tabular} \\
\hline 20 & $\begin{array}{l}\text { CHAPTER } \\
\text { SYSTEMS }\end{array}$ & $\begin{array}{l}21 \text { CHAPTER 3. ANALOG SYSTEMS } \\
\text { Watch video } 5 \\
\text { Questions video } 5 \\
\text { EXERCISES } 1 \text { (Chapter } \mathbf{1 , 2 , 3} \text { ) }\end{array}$ \\
\hline 27 & $\begin{array}{l}\text { EXERCISES } 1 \text { (Chapter 1,2,3) } \\
\text { 1h CHAPTER 4. FUNCTIONAL } \\
\text { BLOCKS (total 3h) }\end{array}$ & \begin{tabular}{|ll}
28 & \\
& \\
CHAPTER 4. FUNCTIONAL BLOCKS \\
CHAPTER 5. AMPLIFIERS WITH \\
TRANSISTORS (total $\mathbf{6 h}$ )
\end{tabular} \\
\hline
\end{tabular}


ANEXO 2

\section{ANALOG ELECTRONICS. ANONYMOUS SURVEY.}

1. Did you watch the videos posted in PoliformaT?
a. Yes, all of them.
b. Most of them (3 or more).
c. A few of them (1 or 2$)$.
d. None of them.

2. What do you think about the time you spent watching the videos?

a. It took me too much time.

b. The investment of time was worth it.

c. I think I saved time because I understood the aspects in the videos and reinforced them in class.

d. It was a waist of time.

3. How did you find the videos?
a. Easy to understand

b. Difficult to follow.

4. How was the rhythm of the videos?
c. The rhythm was too fast.
d. The rhythm was too slow.
e. The rhtyhm was all right.

5. How did you find the written explanations in the videos?
f. Easy to understand

g. Difficult to follow.

6. How did you watch the videos?

a. I stopped and/or went backwards sometimes.

b. Without any stop.

7. How did you find the questions about the videos?

a. They were easy to solve.

b. I had some trouble solving the questions.

8. How did you like the method of watching videos in chapter 3 ?

a. I liked the method used in chapter 3, I think the videos helped to understand the theoretical aspects and we had more time to work in class on the practical aspects.

b. I don't think the videos are a good system to assimilate the theoretical aspects and prefer the explanations made in class, as in the rest of the chapters.

9. The methodological distribution of analog electronics should be:

a. All chapters should have videos. Although this would require investment of time at home, the student can take more profit of the class sessions based on exercises and discussions based on the video contents.

b. I prefer the traditional teaching, with no videos at all.

c. Only one chapter with videos is OK.

d. $50 \%$ of the chapters should use the videos methodology.

10. Free suggestions to improve the teaching methods based on videos:

11. Other suggestions to improve teaching methods in analog electronics classes: 